J. Gunn

T. W. Harding

M. Harper

G. Harrison

J. Harrison

F. Hassanyeh

K. Hawton

J. H. Hendriks

P. Hill

P. Hoare

F. Holloway

J. Holmes

A. O. House

R. Howard

P. Howorth

P. Hughes

A. James

C. Johnson

D. Johnson

S. Johnston

D. Jolley

P. Jones

P. Joseph

J. Joyce

E. Kapp

A. Kendrick

H. Kennedy

P. F. Kennedy

A. Kent

D. I. Khoosal

B. Kidd

D. Kingdon

S. R. Kisely

N. Kreitman

H. Lacey

M. Launer

R. Leetham

J. Leff

P. Lelliot

F. Lieh-Mak

A. Lillywhite

J. Lindesay
M. Lipsedge

R. Littlewood

G. G. Lloyd

R. Lyall

S. Marriott

M. Marshall

A. Maden

G. Masterson

R. Mayou

S. McCluskey

K. McKenzie

D. McLean

E. Mendelson

S. Milne

P. Misch

M. Mitcheson

M. Monaghan

H. G. Morgan

D. Mumford

D. Myers

A. R. Nicol

A. Nikapota

M. P. Nowers

C. Nunn

C. M. O'Driscoll

S. Onyett

M. Orrell

O. A. Oyebode

D. A. Patience

W. Parry-Jones

T. Pastor

R. Peveler

M. Phelan

R. M. Philpott

L. Pilowsky

B. Pitt

M. Prendergast

K. Pugh

G. Pullen

L. Ratna

P. Raven

A. Reid
J. Reilly

L. Riflin

J. Rigby

H. Rollin

N. Rose

P. Sashidharan

J. Scott

R. Seifert

M. Serfaty

C. Sharp

M. S. Shooter

M. Silverman

I. Skeldon

P. Snaith

P. Snowden

C. Stark

J. Stern

G. Strathdee

P. Sugarman

K. Sutherby

D. Taylor

E. Taylor

P. J. Taylor

R. Tillett

C. Thompson

G. Thornicroft

S. P. Tyrer

J. van Os

C. Van Velsen

M. Venning

G. G. Wallis

R. Warner

J. P. Wattis

M. P. I. Weller

S. C. Wessely

P. Whewell

Y. Wiley

D. Will

S. Wilson

A. Wright

T. Wykes

A. J. Yellowlees

\title{
Study tour for College Members
}

A study tour to Normandy is planned to take place in May 1996. The outline is as follows:

- Saturday, 18th May. Depart London by coach to Caen, France.

- 20th and 21st - Visit hospitals in Caen.

- 22nd - Joint presentation of papers.
- 23rd and 24th - Visit hospitals in Rouen.

- Return by coach on Saturday, 25th May.

Further information available from Dr Cyril Davies, Hillview Lodge, Royal United Hospital, Combe Park, Bath BAl 3NG. (Tel: 01225825361) 